\title{
Echinodermata da Praia do Cabo Branco, João Pessoa, Paraíba, Brasil
}

\author{
Anne Isabelley Gondim ${ }^{1,3}$, Patrícia Lacouth ${ }^{1}$, Carmen Alonso ${ }^{1}$ \& Cynthia Lara de Castro Manso ${ }^{2}$ \\ ${ }^{1}$ Laboratório de Invertebrados Paulo Young, \\ Departamento de Sistemática e Ecologia, Campus I, \\ Universidade Federal da Paraíba - UFPB, \\ Cidade Universitária, CEP 58051-900, João Pessoa, PB, Brasil \\ ${ }^{2}$ Laboratório de Invertebrados Marinhos, Campus Prof. Alberto Carvalho, \\ Universidade Federal de Sergipe - UFS, \\ Rua Olimpo Grande, s/n, Sitio Porto, CEP 49500-000, Itabaiana, SE, Brasil \\ ${ }^{3}$ Autor para correspondência: Anne Isabelley Gondim, e-mail: anneisabelley@yahoo.com.br
}

GONDIM, A.I., LACOUTH, P., ALONSO, C. \& MANSO, C.L.C. 2008. Echinodermata from Cabo Branco Beach, João Pessoa, Paraíba, Brazil. Biota Neotrop. 8(2): http://www.biotaneotropica.org.br/v8n2/en/abstrac t?inventory+bn02408022008.

\begin{abstract}
The present study examined the fauna of echinoderms at Cabo Branco Beach, João Pessoa, state of Paraíba, Brazil. The beach was formed by the erosion of a sandstone terrace. Thirty two species from four echinoderm classes (Asteroidea, Ophiuroidea, Echinoidea and Holothuroidea) distributed among 18 families and 24 genera were identified. The number of identified taxons corresponds to $9.7 \%$ of the total number of species registered for the entire Brazilian coast, and demonstrates the significant diversity of echinoderm species in this area. Class Ophiuroidea was the most diverse, with 13 species, followed by Holothuroidea (10 spp.), Asteroidea (5 spp.) and Echinoidea (4 spp.). Among the recorded species, the following were new occurrences for the state of Paraíba: Ophiocnida scabriuscula, Ophiomyxa flaccida, Ophiactis lymani, Ophionereis reticulata, Ophioderma januarii, Ophiophragmus brachyatis, Thyone cognita, T. suspecta, Ocnus suspectus, Phyllophorus occidentalis, P. communis, Lissothuria sp., Holothuria grisea, Holothuria arenicola, Chiridota rotifera, Synaptula hydriformis, Echinaster nudus, Linckia guildingii, Lytechinus variegatus, Mellita quinquiesperforata and Leodia sexiesperforata.
\end{abstract}

Keywords: Echinoderms, distribution, biodiversity, northeastern Brazil.

GONDIM, A.I., LACOUTH, P., ALONSO, C. \& MANSO, C.L.C. 2008. Echinodermata da Praia do Cabo Branco, João Pessoa, Paraíba, Brasil. Biota Neotrop. 8(2): http://www.biotaneotropica.org.br/v8n2/pt/abstra ct?inventory+bn02408022008.

Resumo: Este estudo retrata a fauna de equinodermos encontrada na Praia do Cabo Branco, João Pessoa, Paraíba, Brasil. Esta praia é formada por um terraço de abrasão marinha do tipo arenito-ferruginoso e está localizada no ponto mais oriental das Américas. Foram identificadas 32 espécies de equinodermos pertencentes a quatro classes (Asteroidea, Ophiuroidea, Echinoidea e Holothuroidea), distribuídas em 18 famílias e 24 gêneros. O número de táxons identificados corresponde a 9,7\% das espécies registradas para o litoral Brasileiro e mostra a significativa diversidade das espécies de Echinodermata encontrada nesta área. A classe Ophiuroidea foi a mais diversa com 13 espécies, seguida por Holothuroidea (10 spp.), Asteroidea (5 spp.) e Echinoidea (4 spp). Do total de espécies registradas, as seguintes são novas ocorrências para o litoral do Estado da Paraíba: Ophiocnida scabriuscula, Ophiomyxa flaccida, Ophiactis lymani, Ophionereis reticulata, Ophioderma januarii, Ophiophragmus brachyatis, Thyone cognita, T. suspecta, Ocnus suspectus, Phyllophorus occidentalis, P. communis, Lissothuria sp. Holothuria grisea, H. arenicola, Chiridota rotifera, Synaptula hydriformis, Echinaster nudus, Linckia guildingii, Lytechinus variegatus, Mellita quinquiesperforata e Leodia sexiesperforata.

Palavras-chave: Equinodermos, distribuição, biodiversidade, nordeste do Brasil. 


\section{Introdução}

O Filo Echinodermata é um dos grupos de maior importância na estrutura das comunidades bentônicas marinhas, pois inclui organismos que ocupam diversos nichos ecológicos. São encontrados em todos os oceanos, tanto nas zonas polares quanto tropicais, desde a região do mesolitoral até as grandes profundidades (Hyman 1955, Hadel et al. 1999, Ventura et al. 2007). Nos ambientes tropicais, os equinodermos estão entre os organismos mais abundantes e diversos, e ocorrem em substratos consolidados ou não, e até mesmo em epibiose com outros animais ou plantas (Hyman 1955, Hendler et al. 1995, Hadel 1997). No Brasil são conhecidas mais de 300 espécies, enquanto que no mundo existem cerca de 7.000 espécies viventes (Pawson 2007). Nas comunidades marinhas os equinodermos desempenham importantes papéis ecológicos (Benitez-Villalobos 2001), pois muitos são capazes de controlar as densidades populacionais de determinadas espécies (Ventura et al. 2006), atuam na reciclagem de nutrientes e nos processos bioerosivos (Hadel et al. 1999).

Os primeiros estudos sobre os Echinodermata no Brasil sempre tiveram um caráter de avaliação geral (Hadel et al. 1999), citando a ocorrência de espécies ao longo de todo o litoral brasileiro. Para as regiões sul e sudeste são conhecidos diversos trabalhos enfocando o grupo como um todo (Krau 1950, Brito 1971, Hadel et al.1999, Netto et al. 2005, Ventura et al. 2006, 2007), bem como estudos mais concentrados em classes específicas (Tommasi 1970b, Manso 1988a, b, c, d, 1989, 1993, Manso \& Absalão 1988, Borges et al. 2002, Borges \& Amaral 2006 - Ophiuroidea, Tommasi 1965, 1969b, MacCord \& Duarte 2002 - Crinoidea, Tommasi 1969a, Tiago \& Ditadi 2001, Mendes et al. 2006 - Holothuroidea, Tommasi 1966, Tavares \& Borzone 2006 - Echinoidea e Tommasi 1970a Asteroidea). O conhecimento sobre a fauna de equinodermos na Região Nordeste ainda é considerado escasso, principalmente nos estados do Piauí, Maranhão, Paraíba e Alagoas. Para estas áreas não existem publicações específicas sobre a diversidade local do filo, apenas citações da ocorrência de espécies em trabalhos de caráter mais geral realizados no litoral brasileiro, como o de Rathbum (1879), Lima-Verde (1969), Tommasi (1966, 1970a, b), Young (1986) e Albuquerque \& Guille (1991).

No entanto publicações específicas sobre o grupo são encontradas em alguns estados do Nordeste. No Ceará Martins \& Martins de Queiroz (2006) realizaram um levantamento das espécies de equinodermos encontradas na costa oeste deste Estado. Em Pernambuco, Fernandes et al. (2002) inventariaram os Echinodermata do litoral pernambucano e Neves et al. (2007) estudaram os ofiuróides associados ao octocoral Carijoa riisei. Em Sergipe, Manso \& Farias (1999) registraram a ocorrência de ofiuróides no conteúdo gastrointestinal do baiacu Sphoeroides testudineus. E na Bahia, Manso (1993, 2004) estudou respectivamente os ofiuróides do sul da Bahia e bancos submarinos adjacentes e os equinodermas da Bacia de Camamu, Alves \& Cerqueira (2000) realizaram um levantamento das espécies de equinodermos encontrados em cinco praias de Salvador, e Magalhães et al. (2005) fizeram uma lista de espécies para o estado, com base em dados bibliográficos.

O objetivo do presente trabalho é inventariar a fauna de Echinodermata encontrada na praia de Cabo Branco, localizada na região metropolitana de João Pessoa, no Estado da Paraíba com considerações sobre os seus hábitats.

\section{Material e Métodos}

\section{1. Área de estudo}

O material examinado é proveniente da Ponta do Cabo Branco $\left(07^{\circ} 08^{\prime} 50^{\prime \prime} \mathrm{S}\right.$ e $\left.34^{\circ} 47^{\prime} 51^{\prime \prime} \mathrm{W}\right)$, que está situada ao sul da Praia do
Cabo Branco, João Pessoa, Paraíba, Brasil (Figura 1) e localiza-se no ponto mais oriental das Américas (Feliciano \& Mélo 2003). A ponta do Cabo Branco possui aproximadamente $1,16 \mathrm{~km}$ de extensão e caracteriza-se por apresentar um terraço de abrasão marinha margeado por uma falésia (Figura 2), o qual é composto por aglomerados de rochas arenito-ferruginosas que se estendem por centenas de metros mar adentro. Estes formam parte dos recifes paralelos à linha de costa, que juntamente com a variada disposição das rochas e dos sedimentos, formam diversos micro-habitats para a fixação de várias espécies.

Trata-se de uma área alvo de vários estudos, entre os quais se destacam: Rosa et al. (1997) estudaram a diversidade da ictiofauna de poças de maré, Gama et al. (2001) fizeram um estudo sazonal das comunidades de substrato duro, Gama et al. (2006) analisaram o recrutamento e colonização das ascídias em rochas e Barbosa \& Leonel (2003) estudaram a comunidade de crustáceos associados a algas na região entre-marés. Por estar localizada dentro do perímetro urbano esta região sofre forte ação antrópica (por exemplo, a pesca e o turismo).

\section{Material examinado}

Para confeccionar a lista de espécies foram identificados os lotes depositados na Coleção de Invertebrados Paulo Young do Departamento de Sistemática e Ecologia da Universidade Federal da Paraíba (CIPY-DSE-UFPB), que inclui exemplares coletados na área de estudo entre 1978 e 2007 . As considerações sobre os hábitats foram inferidas a partir de informações contidas nas etiquetas de tombo e por observações feitas em campo.

Para a identificação das espécies foram utilizadas as chaves taxonômicas de Deichmman (1930), Tommasi (1966, 1969, 1970a, b) como também as descrições de Thomas (1973), Manso (1988d), Clark \& Downey (1991), Hendler et al. (1995) e Borges et al. (2002).

\section{Resultados}

Foram identificadas 32 espécies pertencentes a quatro classes do filo Echinodermata distribuídas em nove ordens, 18 famílias e 24 gêneros (Tabela 1). A classe com a maior número de espécies foi Ophiuroidea $(\mathrm{n}=13)$, seguida de Holothuroidea $(\mathrm{n}=10)$, Asteroidea $(n=5)$ e Echinoidea $(n=4)$. Os exemplares foram registrados em sua maioria sob rochas, enterrados no sedimento, como também nos fitais de Halimeda opuntia, Sargassum polyceratium, Lithothamnium sp., Ulva lactuta, Hypnea musciformis, Padina gymnospora e Gracilaria caudata (Tabela 1).

Do total de espécies registradas são novas ocorrências para o litoral do Estado da Paraíba: Ophiocnida scabriuscula, Ophiomyxa flaccida, Ophiactis lymani, Ophionereis reticulata, Ophioderma januarii, Ophiophragmus brachyatis, Thyone cognita, T. suspecta, Ocnus suspectus, Phyllophorus occidentalis, P. communis, Lissothuria sp., Holothuria grisea Figura 3b, H. arenicola, Chiridota rotifera, Synaptula hydriformis, Echinaster nudus, Linckia guildingii, Lythechinus variegatus, Mellita quinquiesperforata e Leodia sexiesperforata.

Os indivíduos da classe Asteroidea foram registrados sobre rochas e enterrados no sedimento, sendo Astropecten marginatus e Luidia senegalensis citadas para fora do terraço de abrasão.

Os espécimes da classe Ophiuroidea registrados do fital eram, em sua maioria, juvenis e exclusivamente representantes das famílias Amphiuridae, Ophiactidae e Ophiothrichidae, enquanto os indivíduos das famílias Ophionereididae e Ophiodermatidae Figura 3a foram citados apenas sob pedras, sendo todos os indivíduos adultos.

Na classe Echinoidea, a espécie Echinometra lucunter (Figura 3f) foi registrada tanto em locas de rochas como sob estas, enquanto Lytechinus variegatus (Figura 3e) foi encontrada sobre a areia e 

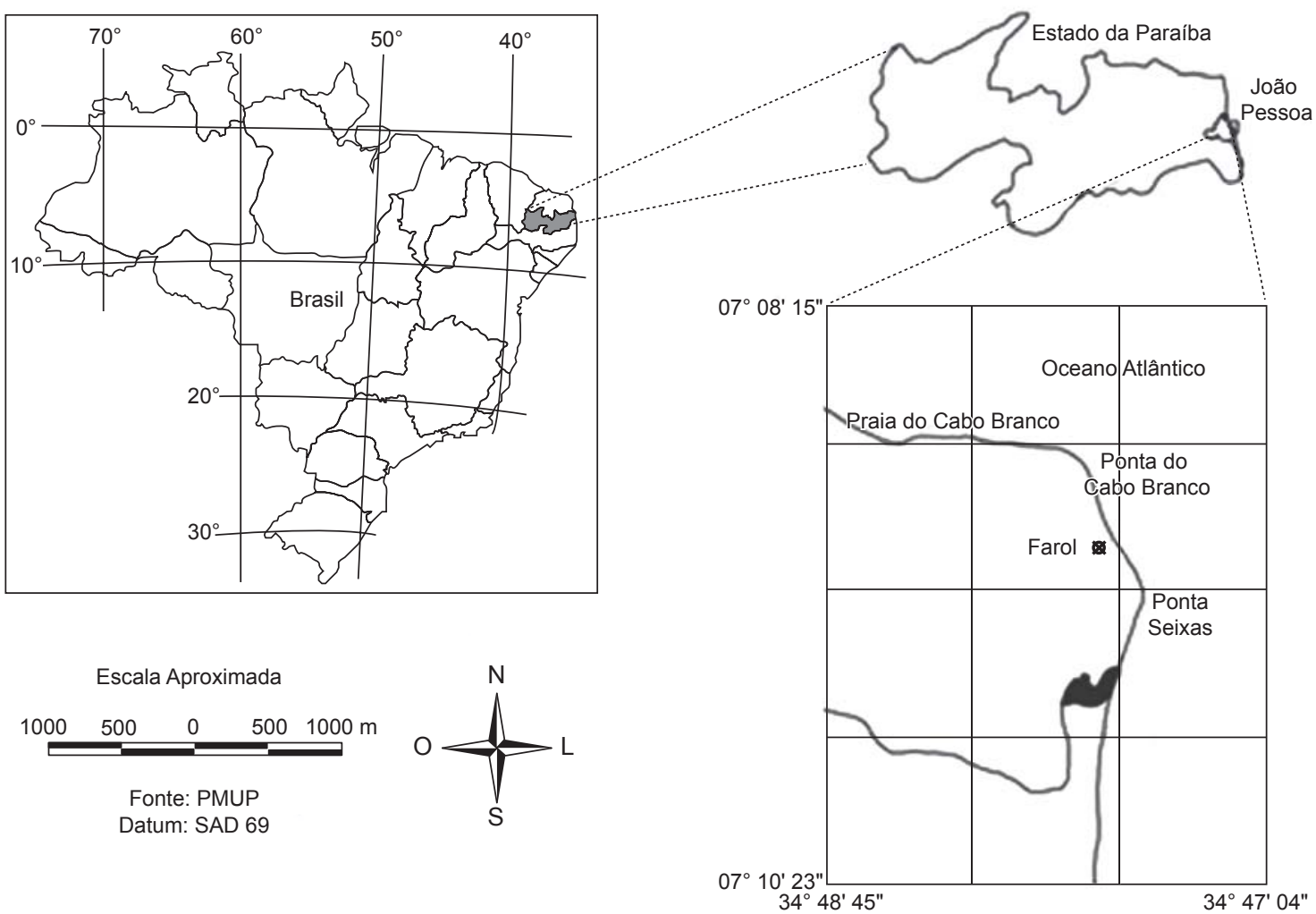

Figura 1. Localização geográfica da área de estudo, Cabo Branco, na costa do Estado da Paraíba (modificado de Gama 2006).

Figure 1. Geographic location of the Cabo Branco in the coast of Paraiba State (modified of Gama 2006).

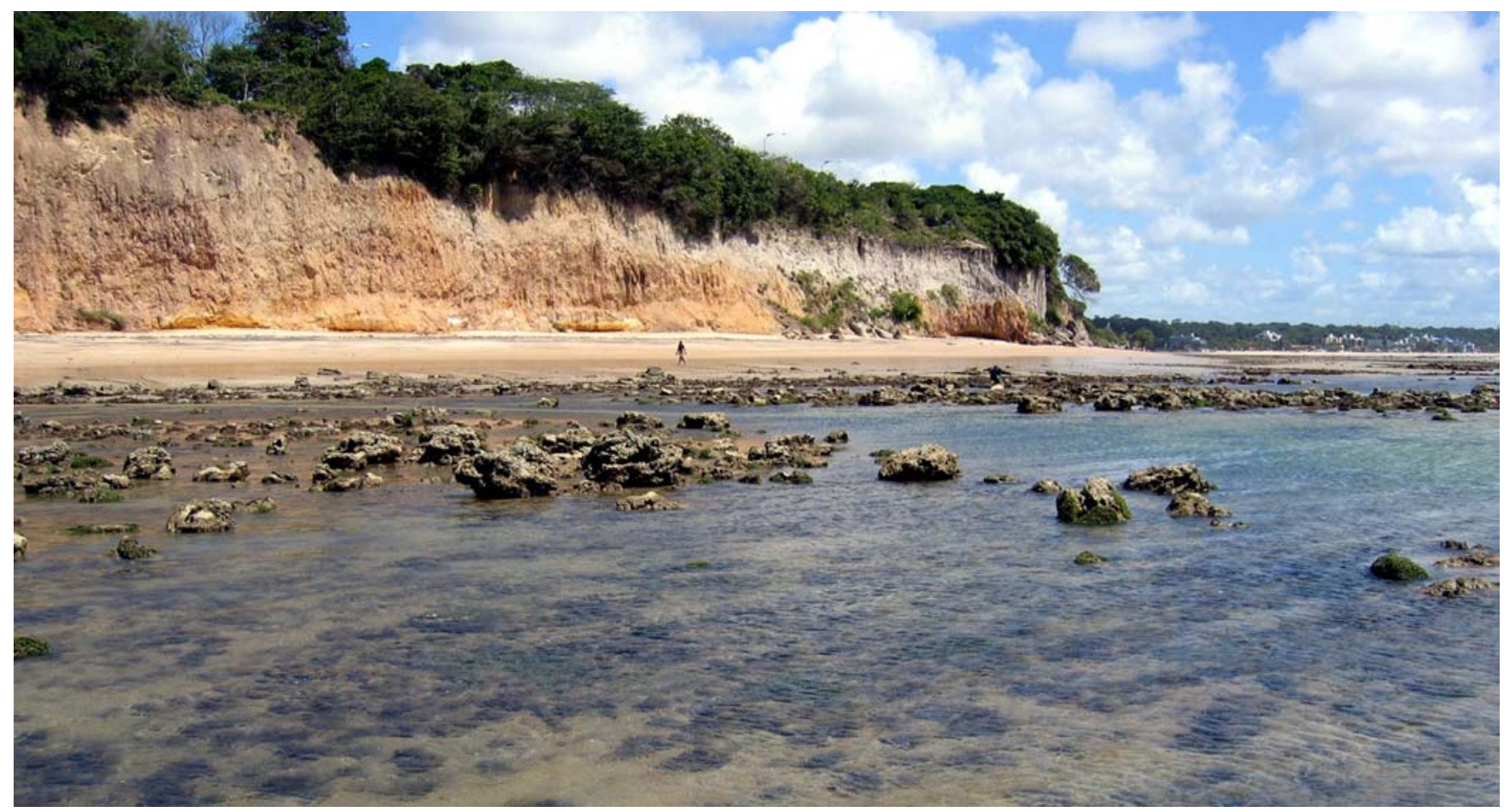

Figura 2. Vista parcial dos recifes do Cabo Branco, João Pessoa, Paraíba (Foto: Thelma Dias).

Figure 2. Partial view of the Cabo Branco reefs, João Pessoa, Paraíba (Photo: Thelma Dias). 
Tabela 1. Lista sistemática das espécies de Echinodermata registradas na Praia do Cabo Branco, João Pessoa, Paraíba. Table 1. Systematic list of the echinoderms species registered at the Cabo Branco Beach, João Pessoa, Paraíba.

$\begin{array}{lll}\text { Classe } & \text { Família } & \text { Espécie } \\ \text { Ordem } & & \end{array}$

Asteroidea De Brainville, 1830
Spinulosida Perrier, 1884
Valvatida Perrier, 1884
Paxillosida Perrier, 1884
Ophiuroidea Gray, 1840
Phrynophiurida Matsumoto, 1915
Ophiurida Muller \& Troschel, 1840

Ophiurida Muller \& Troschel, 1840

Echinoidea Leske, 1867

Echinoida Claus, 1876

Clypeasteroida Agassiz, 1873

Holothuroidea de Blainville, 1834

Dendrochirotida Grube, 1840

Aspichirotida Grube, 1840

Apoda Brandt, 1835
ECHINASTERIDAE Verrill, 1867

OPHIDIASTERIDAE Verrill, 1867

LUIDIIDAE Verrill, 1889

ASTROPECTINIDAE Gray, 1840

OPHIOMYXIDAE Ljungman, 1867

AMPHIURIDAE Ljungman, 1867

OPHIACTIDAE Matsumoto, 1915

OPHIONEREIDIDAE Ljungman, 1867

OPHIODERMATIDAE

Ljungman, 1867

OPHIOTHRICHIDAE Ljungman, 1866

ECHINOMETRIDAE Gray, 1825

TOXOPNEUSTIDAE (Troschel, 1872)

MELLITIDAE Stefanini, 1911

CUCUMARIIDAE Perrier, 1902

PHYLLOPHORIDAE Bell, 1884

HOLOTHURIIDAE Ludwig, 1894

CHIRIDOTIDAE Oestergren, 1898
Echinaster echinophorus (Lamarck, 1816)

Echinaster nudus Tommasi, 1970

Linckia guildingii Gray, 1840

Luidia senegalensis (Lamarck, 1816)

Astropecten marginatus Gray, 1840

Ophiomyxa flaccida (Say, 1825)

Amphipholis januarii Ljungman, 1867

Amphipholis squamata (Delle Chiaje, 1828)

Ophiocnida scabriuscula (Lütken, 1859)

Ophiophragmus brachyatis Clark, 1915

Amphiodia gracilima (Stimpson, 1852)

Ophiactis lymani (Ljungman, 1872)

Ophiactis savignyi (Müller \& Troschel, 1842)

Ophionereis reticulata (Say, 1825)

Ophioderma appressum (Say, 1825)

Ophioderma cinereum Müller \& Troschel,1842

Ophioderma januarii (Lütken, 1856)

Ophiothrix (Ophiothrix) angulata (Say, 1825)

Echinometra lucunter (Linnaeus, 1758)

Lytechinus variegatus (Lamarck, 1816)

Mellita quinquesperforata (Leske, 1778)

Leodia sexiesperforata (Leske, 1778)

Thyone cognita (Cherbonnier, 1961)

Thyone suspecta Ludwig, 1874

Ocnus suspectus Ludwig, 1874

Lissothuria sp.

Phyllophorus occidentalis (Ludwig, 1885)

Phyllophorus comunis (Forbes, 1841)

Holothuria grisea Selenka, 1867

Holothuria arenicola Semper, 1868

Chiridota rotifera (Pourtalés, 1851)

Synaptula hydriformis (Lesueur, 1851)

*Os números sobrescritos correspondem às algas onde foram encontradas as espécies de Echinodermata: ${ }^{1}$ Halimeda opuntia (Linnaeus) J. V. Lamouroux, 1816; ${ }^{2}$ Ulva lactuta Linnaeus, $1753 ;{ }^{3}$ Lithothamnium sp. R.A. Philippi; ${ }^{4}$ Gracilaria caudata J. Agardh, $1852 ;{ }^{5}$ Padina gymnospora (Kützing) Sonder, 1871;

${ }^{6}$ Sargassum polyceratium Montagne, 1837 e ${ }^{7}$ Hypnea musciformis (Wulfen) J. Lamouroux, 1813. 


\begin{tabular}{|c|c|c|c|c|}
\hline \multirow[t]{2}{*}{$\begin{array}{l}\text { Sob } \\
\text { rochas }\end{array}$} & \multirow[t]{2}{*}{$\begin{array}{l}\text { Sobre } \\
\text { rochas }\end{array}$} & \multirow[t]{2}{*}{ Fital } & $\begin{array}{l}\text { Enterrado no } \\
\text { sedimento }\end{array}$ & \multirow[t]{2}{*}{$\begin{array}{c}\text { Número de Tombo } \\
\text { UFPB-ECH }\end{array}$} \\
\hline & & & Praia Terraço & \\
\hline
\end{tabular}

$139 ; 703 ; 704 ; 706 ; 705 ; 707 ; 708 ; 709 ; 710 ; 711 ; 712 ; 713 ; 714 ; 715 ; 719 ; 722 ; 727 ; 870$ 569

$1159,1160,1161$

1168

1169

$64 ; 65 ; 71 ; 72 ; 291 ; 371$

50;51;53;57;66;374;382;432;698;1107;1108;1109;1032;1150;1088

$55 ; 56 ; 59 ; 63 ; 69 ; 70 ; 473 ; 700 ; 701 ; 941 ; 1117 ; 1151$

298;335;384;390;396;1100;1101;1102;1103 1149;1153

1121

1162

$58 ; 61 ; 73 ; 74 ; 283 ; 284 ; 302 ; 372$

$52 ; 54 ; 60 ; 62 ; 67 ; 68 ; 137 ; 297 ; 301 ; 418 ; 422 ; 1126 ; 1127 ; 1147 ; 1152$

$292 ; 295 ; 299 ; 358 ; 359 ; 378 ; 381 ; 383 ; 398 ; 634 ; 1017 ; 1075$

$117 ; 129 ; 296 ; 336 ; 356 ; 357 ; 377 ; 610$

$118 ; 123 ; 132 ; 333 ; 609$

553

1165

$1 ; 2 ; 3 ; 21 ; 353 ; 354 ; 355 ; 702 ; 1018$

1158 .

1164

1163

$155 ; 1060 ; 1065 ; 1066$

882

$1061 ; 1063$

1166

866,1067

434, 435, 1064

$207 ; 213 ; 272 ; 276 ; 277 ; 278 ; 311 ; 313 ; 316 ; 319$

$697 ; 1062$

$176 ; 177 ; 178 ; 181 ; 182 ; 185 ; 186 ; 187 ; 188 ; 189 ; 190 ; 191 ; 192 ; 193 ; 194 ; 195 ; 196 ; 198 ;$ $199 ; 200 ; 853 ; 1068$ 

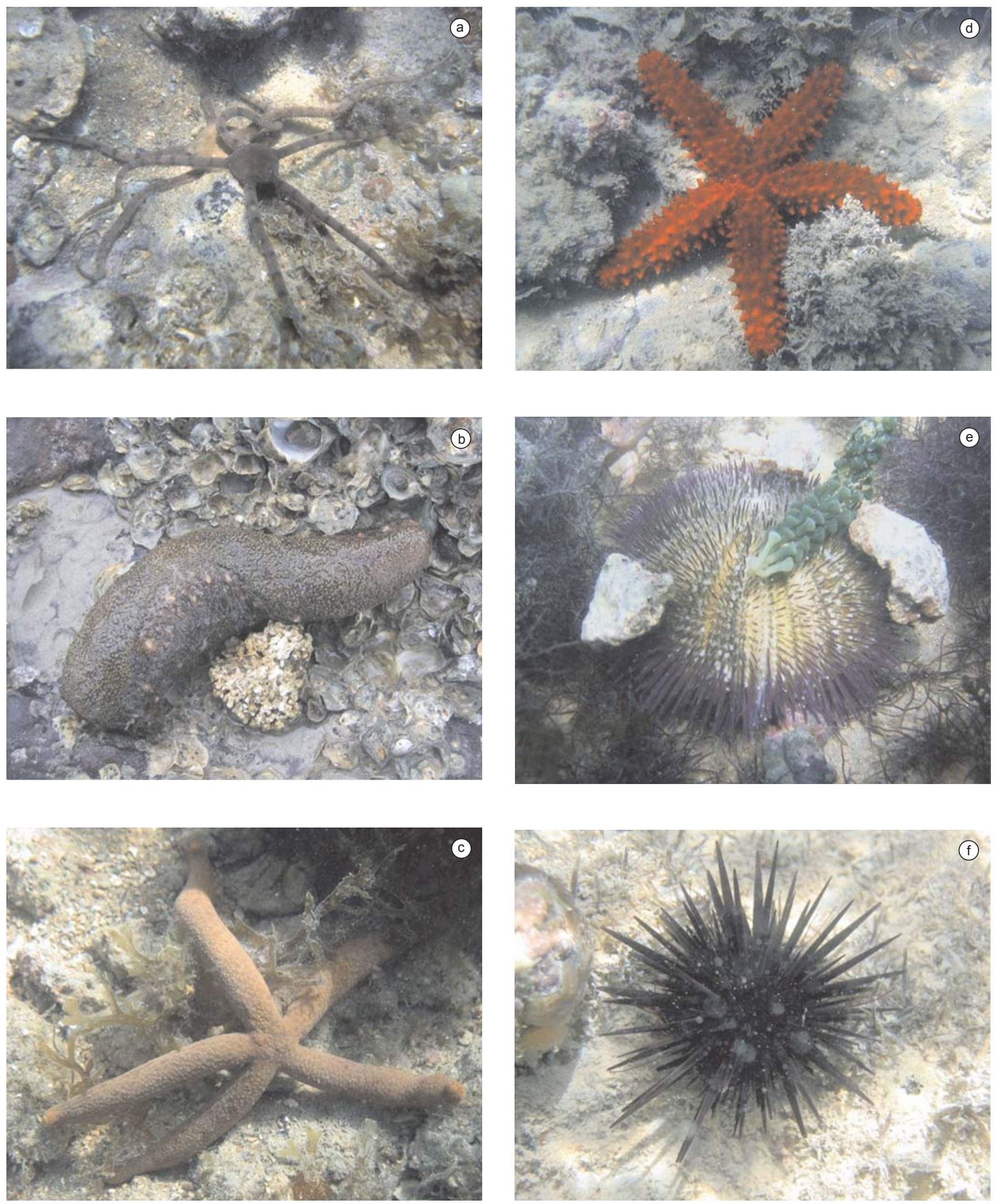

Figura 3. Algumas espécies de equinodermos registrados no Cabo Branco, João Pessoa, Paraíba: a) Ophioderma cinereum (25 mm de diâmetro do disco), b) Holothuria grisea (20 cm de comprimento total), c) Linckia guildingii (12 cm de raio maior), d) Echinaster echinophorus (10 cm de raio maior), e) Lytechinus variegatus (12 cm de diâmetro) e f) Echinometra lucunter (10 cm de diâmetro) (Fotos: Thelma Dias).

Figure 3. Some of the echinoderms species found at Cabo Branco, João Pessoa, Paraíba: a) Ophioderma cinereum (25 mm in disc diameter), b) Holothuria grisea (20 cm in total length), c) Linckia guildingii (12 cm in major radii), d) Echinaster echinophourus (10 cm in major radii), e) Lytechinus variegatus (10 $\mathrm{m}$ in diameter) and f) Echinometra lucunter (10 cm in diameter) (Photos: Thelma Dias). 
geralmente recoberta por fragmentos de conchas de bivalves, de bolachas-do-mar, de algas, e outros. Mellita quinquesperforata e Leodia sexiesperforata foram observadas enterradas no sedimento, com uma fina camada de areia sobre as mesmas.

Os indivíduos da classe Holothuroidea foram observados freqüentemente sob rochas e em fendas. Apenas Synaptula hydriformis ocorreu associada aos fitais de Halimeda opuntia e Lithothamnium sp. Chiridota rotifera foi tipicamente observada enterrada em sedimentos finos, sob rochas e vivendo de forma agregada.

\section{Discussão}

Ao se comparar o número de espécies de equinodermos registradas no mundo (7.000) com as do Brasil (300) é possível perceber o número reduzido de estudos realizados sobre o grupo no litoral brasileiro, tendo em vista que se trata de organismos amplamente distribuídos e diversos.

O número de espécies encontradas na área estudada corresponde a $9,7 \%$ do total registrado para o Brasil, representando um grande número considerando o tamanho da área (1,16 km de extensão). Esta diversidade se deve, provavelmente, a vários fatores como, por exemplo, os diferentes hábitats e tipos de substratos, como areia grossa, areia lodosa e cascalho (Lana et al. 1996), além de uma grande diversidade de flora. A complexidade deste ambiente pode torná-lo favorável aos equinodermos, pois estes ocupam diferentes nichos dentro do ecossistema (Lawrence 1987).

A Ponta do Cabo Branco mostrou uma fauna de equinodermos bem diversificada e tipicamente tropical, na qual a classe Ophiuroidea foi a mais representativa. Este resultado assemelha-se aos obtidos por Martins \& Martins de Queiroz (2006), Alves \& Cerqueira (2000) e Lima-Verde (1969), para alguns estados da região Nordeste. De acordo com Tommasi (1966, 1969, 1970a, b) as espécies de Echinodermata registradas no presente estudo são tipicamente litorâneas, ou possuem ampla distribuição batimétrica.

Dentre os Asteroidea, a espécie com maior número de registros foi Echinaster echinophorus (Figura 3d) que é uma espécie típica do litoral brasileiro (Hendler et al. 1995). De acordo com Bernasconi (1958) era uma espécie comum para o estado da Paraíba, porém nos dias atuais é visualmente pouco numerosa. A espécie Linckia guildingii (Figura 3c) foi registrada sobre rochas, no entanto de acordo com Hendler et al. (1995) ela também pode ser encontrada em bancos de areia. Segundo Brito (1971) é uma espécie abundante no nordeste brasileiro, porém sua ocorrência ao longo do litoral nordestino ainda não está completamente conhecida. A maioria dos exemplares desta espécie possui um braço mais longo e outros menores em processo de regeneração, o que pode estar relacionado ao fato desta espécie se reproduzir predominantemente de forma assexuada (Hyman 1955). Astropecten marginatus e Luidia senegalensis foram registradas exclusivamente em substratos arenosos onde são predadores da infauna de moluscos destes ambientes (Hyman 1955). Todos os representantes da classe Asteroidea citados, exceto Echinaster nudus, estão incluídos na lista brasileira de espécies ameaçadas de extinção (MMA 2004).

Com relação aos Ophiuroidea, a alta diversidade observada pode estar relacionada à capacidade desses animais viverem em hábitats variados. Os espécimes encontrados nos fitais eram em sua maioria indivíduos jovens, o que sugere que este ambiente pode ser utilizado como local de recrutamento. Porém também o utilizam de forma permanente, como é o caso de Ophiactis savignyi que foi observado em vários estádios de vida neste tipo de ambiente. Amphipholis squamata é uma espécie vivípara (Irimura 1982), encontrada associada a algas e outros tipos de substratos biológicos. Neste estudo foram observados apenas indivíduos juvenis no fital, o que sugere que possivelmente esta espécie deposita sua prole neste ecossistema, por este representar um local protegido. No entanto, estudos sobre o comportamento reprodutivo desta espécie devem ser realizados para que seja avaliado como esta espécie utiliza o fital.

As espécies das famílias Ophiodermatidae e Ophionereididae são mais comuns embaixo de pedras, sob as quais também se encontra um grande número de espécies de ascídias, esponjas e poliquetas. Considerando que os Ophiodermatidae possuem hábitos alimentares extremamente variados, podemos sugerir que eles utilizam tanto as espécies que ali vivem como também os indivíduos da zona intersticial como alimento. Além disso, as duas famílias utilizam este ambiente para se protegerem de predadores e da luminosidade (Hyman 1955).

Echinometra lucunter é a espécie visualmente mais comum na área de estudo, vivendo em locas e principalmente na região do mesolitoral que fica o maior tempo exposta. A grande abundância desta espécie no ambiente sugere que não devam existir muitos predadores ou parasitas que consigam controlar a população do ouriço, como também, que se trata de uma espécie extremamente resistente a períodos de ressecamento e a mudanças de temperatura e salinidade (Hendler et al. 1995). Lytechinus variegatus é uma espécie aparentemente rara no local, sendo encontrada próxima a rochas no infralitoral e com fragmentos recobrindo o corpo devido a sua sensibilidade à luz. As espécies Mellita quinquesperforata e Leodia sexiesperforata ocorrem em baixo número e restritas às regiões com sedimento arenoso dentro e fora do terraço de abrasão marinha.

A diversidade encontrada na classe Holothuroidea foi bastante representativa no Cabo Branco, comparando-se com os resultados apresentados por Alves \& Cerqueira (2000) que encontraram cinco espécies nas praias de Salvador, e Martins \& Martins de Queiroz (2006) que registraram apenas uma espécie nas praias do Ceará. As nove espécies de Holothuroidea registradas na Ponta do Cabo Branco são típicas de regiões tropicais de águas quentes e litorâneas (Tommasi 1969). Chiridota rotifera é a espécie mais comum da área, possivelmente devido à presença de biodetritos, considerado o habitat preferencial desta espécie (Hadel 1997). Esta espécie é frequentemente encontrada em agregados de 10 a 15 indivíduos, coincidindo com as observações de Hadel (1997) e Martins \& Martins de Queiroz (2006).

A partir dos resultados deste trabalho conclui-se que nesta pequena área, os Echinodermata, especialmente os Ophiuroidea e Holothuroidea, podem ser considerados um grupo bem representado. Estes dados contrastam com os estudos de Martins \& Martins de Queiroz (2006) que encontraram apenas 12 espécies em seis praias do litoral Cearense, assim como Alves \& Cerqueira (2000) que registraram 28 taxa em cinco praias do litoral Bahiano, e Lima-Verde (1969) que notificou 31 espécies para a costa nordestina.

Ressaltamos que, estudos de cunho ecológico devem ser realizados para uma avaliação da preferência de habitat, da riqueza e da estrutura populacional destes organismos, tendo em vista que a Ponta do Cabo Branco encontra-se ameaçada pela construção de obras de engenharia, que pretendem controlar o processo natural da erosão da falésia e pela intensa exploração dos recursos naturais (como a pesca, o turismo, coleta de souvenires, etc.). Estes impactos, se continuados, poderão modificar toda esta comunidade, que possivelmente sofrerá uma diminuição notória na sua diversidade de espécies.

\section{Agradecimentos}

Agradecemos à $\mathrm{Dr}^{\mathrm{a}}$ Rosa Maria Veiga Leonel por autorizar a utilização do material do fital, à Dra Thelma L. Dias pela leitura criteriosa do texto e valiosas sugestões, ao Dr. Martin Lindsey Christorffersen pela correção do abstract e a todos que de alguma forma contribuíram na realização deste trabalho. 


\section{Referências Bibliográficas}

ALBUQUERQUE, M.N. \& GUILLE, A. 1991. Ophiuroidea (Echinodermata) ao largo do Brasil: Bancos dos Abrolhos, Cadeia Submarina VitóriaTrindade e Plataforma Continental adjacente. Bol. Mus. Nac., N. S., Zool. 353: 1-29.

ALVES, O.F.S. \& CERQUEIRA, W.R P. 2000. Echinodermata das praias de Salvador (Bahia, Brasil). Rev. Bras. Zool. 17(2): 543-553.

BARBOSA, J.H.A.J. \& LEONEL, R.M.V. 2003. Sobre a comunidade de crustáceos em algas de entre-marés: Uma ênfase aos Brachyura. Cad. Camilliani Rev. Faculd. São Camilo Espírito Santo 4(1): 19-29.

BENITEZ-VILLALOBOS, F. 2001. Comparación de la comunidad de Equinodermos, associada a arrecifes, en dos localidades de las Bahías de Huatulco, Oaxaca, México. Cienc. Mar 5(13): 19-24.

BERNASCONI, I. 1958. Asteroideos de la colección del instituto oceanográfico de la Universidad de San Pablo. Bol. Inst. Ocenogr. 9(1-2): 13-21.

BRITO, I.M. 1971. Contribuição ao conhecimento dos Echinodermas da Ilha da Trindade, Brasil. Arq. Mus. Nac. 54: 261-265.

BORGES, M., MONTEIRO, A.M.G. \& AMARAL, A.C. 2002. Taxonomy of Ophiuroidea (Echinodermata) from the Continental Shelf and slope of the Southern and Southern Brazilian Coast. Biota Neotrop. 2(2): 1-69 http:// www.biotaneotropica.org.br/v2n2/pt/abstract?article+BN02302022002 (último acesso em 14/03/05).

BORGES, M. \& AMARAL, A.C. 2006. Filo Echinodermata. In Manual de identificação dos invertebrados marinhos da região Sudeste-Sul do Brasil (A.C. Amaral, A.E. Rizzo \& E.P. Arruda, eds.). EDUSP, São Paulo, p. 287.

CLARK, A.M. \& DOWNEY, M.E. 1991. Starfishes of the Atlantic. Natural History Museum Publications. Ed. Chapman \& Hall, London, p. 794.

DEICHMANN, E. 1930. The Holothurians of the Western Part of the Atlantic Ocean. Bull Mus Comp. Zool. 71(3): 1-226.

FELICIANO, M.L.M. \& MÉLO, R.B. 2003. Atlas do estado da Paraíba - Informações para a gestão do patrimônio natural. $1^{\text {a }}$ Ed. SEPLAN/ IDEME, João Pessoa, p. 58.

FERNANDES, M.L.B., TOMMASI, L.R. \& LIMA, E.J.B. 2002. Filo Echinodermata de Pernambuco. In Diagnóstico da Biodiversidade de Pernambuco (M. Tabarelli \& J.M.C. Silva, eds.). Massangana, Recife, v. 2 , p. $405-427$.

GAMA, P.B., LEONEL, R.M.V. \& MIRANDA, G.C. 2001. Estudo estacional de comunidade de substrato duro da região de entremarés da Ponta do Cabo Branco, João Pessoa, Paraíba. In: M.F. Souza, M. F. Wanderley (Org.). Iniciados. Sér. Inic. vol 6. João Pessoa, p. 64-84.

GAMA, P.B., LEONEL, R.M.V., HERNÁNDEZ, M.I.M \& MOTHES, B. 2006. Recruitment and colonization of colonial ascidians (Tunicata: Ascidiacea) on intertidal rocks in Northeastern Brazil. Iheringia. Sér. Zool. 96(2): 165-172.

HADEL, V.F. 1997. Comportamento e reprodução de Chiridota rotifera (Echinodermata: Holothuroidea) em laboratório. Tese de Doutorado, Universidade de São Paulo, São Paulo.

HADEL, V.F., MONTEIRO, A.M.G., DITADI, A.S.F., THIAGO, C.G. \& TOMMASI, L.R. 1999. Echinodermata. In Biodiversidade do Estado de São Paulo, Brasil: Síntese do Conhecimento ao Final do Século XX, 3 : Invertebrados Marinhos (A.C. Migotto \& C.G. Thiago, eds.). FAPESP, São Paulo, p. 260-271.

HENDLER, G., MILLER, J.E., PAWSON, D.L. \& KIER, P.M. 1995. Sea stars, sea urchins and allies: echinoderms of Florida and the Caribbean. Smithsonian Institution Press, Washington.

HYMAN, L.H. 1955. The Invertebrates: Echinodermata. The Coelomate Bilateria. McGraw-Hill Book Company, London, v. 4, p.763.

IRIMURA, I. 1982. The Brittle-stars of Samagi Bay. Biological Laboratory Imperial Hourschold, Tokyo, p. 1-95, Figs 1-56, Pls 1-15.

KRAU, L. 1950. Observações sobre os Echinodermas da Baía de Guanabara. Mem. Inst. Oswaldo Cruz 48: 357-362.
LANA, P.C., CAMARGO, M.G. \& BROGIM, R. 1996. O Bentos da costa brasileira: avaliação crítica e levantamento bibliográfico (1858-1996). REVIZEE, MMA/CIRM/FEMAR, Rio de Janeiro, p. 432.

LAWRENCE, J.M. 1987. Functional biology of echinoderms. Croom Helm Ltd., London, p. 340.

LIMA-VERDE, J.S. 1969. Primeira contribuição ao inventário dos Echinodermas do nordeste Brasileiro. Arq. Cienc. Mar. 9(1): 9-13.

MacCORD, F.S. \& DUARTE, L.F.L. 2002. Dispersion in populations of Tropiometra carinata (Crinoidea: Comatulida) in the São Sebastião, São Paulo State, Brazil. Estuar. Coast. Shelf Sci. 54: 19-225.

MAGALHÃES, W.F., MARTINS, L.R. \& ALVES, O.F.S. 2005. Inventário dos Echinodermata do Estado da Bahia. Braz. J. Aquat. Sci. Technol. 9(1): 61-65.

MANSO, C.L.C. 1988a. Sobre a ocorrência de Amphilepis sanmatiensis Bernasconi e D`Agostino, 1975 na costa sul do Brasil (Echinodermata: Ophiuroidea). Rev. Bras. Biol. 48(2): 371-373.

MANSO, C.L.C. 1988b. Uma nova espécie de Ophiactis (Echinodermata: Stelleroidea) da costa sudeste do Brasil. Rev. Bras. Biol. 48(2): 375-379

MANSO, C.L.C. 1988c. Ofiuróides da plataforma continental brasileira. Parte I: Rio de Janeiro. (Echinodermata: Ophiuroidea). Rev. Bras. Biol. 48(4): 845-850.

MANSO, C.L.C. 1988d. Sobre a ocorrência de Amphiura (Ophionema) intricata Lütken, 1869 e Ophiophragmus brachyatis H.L. Clark, 1915 no Brasil (Echinodermata, Ophiuroidea, Amphiuridae). Rev. Bras. Biol. 48(4): 965-968.

MANSO, C.L.C. 1989. Os Echinodermata da plataforma continental interna entre Cabo Frio e Saquarema, Rio de Janeiro, Brasil. Rev. Bras. Biol. 49(2): 355-359.

MANSO, C.L.C. 1993. Ofiuróides da Plataforma Brasileira, Parte II: Norte do Estado do Rio de Janeiro, Estado do Espírito Santo, Sul do Estado da Bahia e bancos Royal Charlotte, Hostpur e Davis (Echinodermata: Ophiuroidea). Rev. Bras. Biol. 53(2): 189-195.

MANSO, C.L.C. \& ABSALÃO R.S. 1988a. Ophiuroidea: Situação Pré Operacional nos sacos de Piraquara, região sob influência da descarga da Central Nuclear Almirante Álvaro Alberto (CNAAA). Rev. Bras. Biol. 48(1): 75-82.

MANSO, C.L.C. \& FARIAS, M.C.V. 1999. Ocorrência de ofiuróides no conteúdo gastrointestinal de "baiacu" Sphoeroides testudineus (Linnaeus, 1758) (Teleostei: Tetraodontidae) no estuário do rio Sergipe (Sergipe). Rev. Nordestina Zool. 2(1): 35-37.

MANSO, C.L.C. 2004. Echinodermata da Bacia de Camamu, Bahia, Brasil. Biol. Geral Exper. 5(1): 19-25.

MARTINS, I.X. \& MARTINS de QUEIROZ, A.C. 2006. Echinodermos do litoral do Estado do Ceará. In Biota Marinha da Costa Oeste do Ceará. Ministério do Meio Ambiente, Brasília, (H. Matthews-Cascon \& T.M.C. Lotufo, eds), p. 199-220.

MENDES, F.M., MARENZI, A.W.C. \& DOMENICO, M.D. 2006. Population patterns and seasonal observations on density and distribution of Holothuria grisea (Holothuroidea: Aspidochirotida) on the Santa Catarina Coast, Brazil. Bull. Becher-de-mer. 23: 5-10.

MMA. Ministério do Meio Ambiente. 2004. Lista Nacional das Espécies de Invertebrados Aquáticos e Peixes Ameaçados de Extinção. Anexo 1. Instrução Normativa Nº5, de 26 de maio de 2004. Disponível em: http:// www.mma.gov.br/port/sbf/fauna/index.html

NETTO, L.F., HADEL, V.F. \& TIAGO, C.G. 2005. Echinodermata from São Sebastião Channel (São Paulo, Bazil). Rev. Biol. Trop. 53(3): 207-218.

NEVES, B.M., LIMA, E.J.B. \& PÉREZ, C.D. 2007. Brittle stars (Echinodermata: Ophiuroidea) associated with the octocoral Carijoa riisei (Cnidaria: Anthozoa) from the littoral of Pernambuco, Brazil. J. Mar. Biol. Ass. U. K. 87(5): 1263-1267.

PAWSON, D.L. 2007. Phylum Echinodermata. Zootaxa. 1668: 749-764.

RATHBUN, R. 1879. A List of the Brazilian Echinoderms, with notes on their distribution, etc. T. Connec. Acad. 5: 139-151. 
ROSA, R.S., ROSA, I.L. \& ROCHA, L.A. 1997. Diversidade da ictiofauna de poças de maré da Praia do Cabo Branco, João Pessoa, Paraíba Brasil. Rev. Bras. Zool. 14(1): 201-212.

TAVARES, Y.A.G. \& BORZONE, C.A. 2006. Reproductive cycle of Mellita quinquiesperforata (Lesk) (Echinodermata, Echinoidea) in two contrasting beach environments. Rev. Bras. Zool. 23(2): 573-580.

THOMAS, L.P. 1973. Western Atlantic Brittlestars of the Genus Ophionereis. Bull. Mar. Sci. Gulf. Caribb. 23(13): 585-599.

TIAGO, C.G. \& DITADI, A.S.F. 2001. Holothurians from Brazilian coast: A historical survey. In Echinoderms 2000 (M. Barker ed.). The Netherlands, Balkema, Lisse, p. 379-384.

TOMMASI, L.R. 1965. Lista dos Crinóides recentes do Brasil. Contrib. Inst. Oceanogr. Univ. São Paulo. Sér. Oceanogr. Biol. 9: 1-33.

TOMMASI, L.R. 1966. Lista dos Echinoides recentes do Brasil. Contrib. Inst. Oceanogr. Univ. São Paulo. Sér. Oceanogr. Biol. 11: 1-50.

TOMMASI, L.R. 1969a. Lista dos Holothuroidea recentes do Brasil. Contrib. Inst. Oceanogr. Univ. São Paulo. Sér. Oceanogr. Biol. 15: 1-50.

TOMMASI, L.R. 1969b. Nova contribuição à lista dos Crinóides recentes do Brasil. Contrib. Inst. Oceanogr. Univ. São Paulo. Sér. Oceanogr. Biol. 17: 1-8.
TOMMASSI, L.R. 1970a. Lista dos Asteróides recentes do Brasil. Contrib. Inst. Oceanogr. Univ. São Paulo. Sér. Oceanogr. Biol. 18:1-161.

TOMMASI, L.R. 1970b. Os Ofiuróides recentes do Brasil e de regiões vizinhas. Contrib. Inst. Oceanogr. Univ. São Paulo. Série Oceanogr. Biol. 20:1-146.

VENTURA, C.R.R., LIMA, R.P.N., NOBRE, C.C., VERÍSSIMO, I. \& ZAMA, P.C. 2006. Filo Echinodermata. In Biodiversidade bentônica da região central da Zona Econômica Exclusiva brasileira (H.P. Lavrado \& B.L. Ignácio, eds.). Museu Nacional, Rio de Janeiro, (Série Livros, 18), p. 389.

VENTURA, C.R.R., VERÍSSIMO, I., NOBRE, C.C. \& ZAMA, P.C. 2007. Filo Echinodermata. In Atlas de invertebrados marinhos da região central da Zona Econômica Exclusiva brasileira, parte 1. (H.P. Lavrado \& M.S. Viana, eds.). Museu Nacional, Rio de Janeiro, (Série Livros, 25), p. 258.

YOUNG, P.S. 1986 Análise qualitativa e quantitativa da fauna associada a corais hermatípicos dos recifes de João Pessoa, Pb. Rev. Bras. Biol. 46(1): 99-126.

Recebido em: 07/02/08 Versão reformulada recebida em: 29/04/08 Publicado em: 16/06/08 